des Eidgenössischen Institutes für Schnee- und Lawinenforschung

Juli 1964

\title{
Anlage zur Untersuchung dynamischer Wirkungen von bewegtem Schnee
}

\author{
Von Bruno SALM
}

(Eidg. Institut für Schnee- und Lawinenforschung, Weißfluhjoch/Davos)

Sonderdruck aus ZAMP 15, 357-375 (1964)

Diese Mitteilung kann durch das Eidgenössische Institut für Schnee- und Lawinenforschun zum Preise von Fr. 3.- bezogen werden 



\title{
Anlage zur Untersuchung dynamischer Wirkungen von bewegtem Schnee
}

\author{
Von Bruno SALM, Eidg. Institut für Schnee- und Lawinenforschung, \\ Weissfluhjoch-Davos
}

\section{Einleitung}

Das Gebiet der Schneemechanik zerfällt in zwei Teilgebiete. Das eine behandelt die Vorgänge in der natürlich abgelagerten Schneedecke. Hier treten nur sehr kleine Geschwindigkeiten auf (Grössenordnung $10^{-3} \mathrm{~m} \mathrm{sec}^{-1}$ ), die Trägheitskräfte sind deshalb gegenüber den Reibungs- und Massenkräften in guter Näherung zu vernachlässigen. Dieses Teilgebiet wurde schon relativ früh in Angriff genommen (HAEFELI, 1934) und im Laufe der Zeit ziemlich ausführlich behandelt [1] [2] [11]'). Das andere Teilgebiet, auf das wir uns im folgenden beschränken, behandelt den Fall, wo sich Schnee vom ursprünglichen Ablagerungsort löst und unter Entwicklung von grossen Geschwindigkeiten (Grössenordnung 10-30 $\mathrm{m} \mathrm{sec}^{-1}$ ) als Lawine zu Tal gleitet. Hier treten neben Reibungs- und Massenkräften massgebend Trägheitskräfte auf.

Obschon auf diesem Gebiet sehr viele qualitative Beobachtungen gemacht wurden, besteht doch ein empfindlicher Mangel an exakten Messungen. Bis anhin wurden lediglich durch natürliche Lawinen auf feste Hindernisse ausgeübte Maximaldrücke sowie einige Lawinengeschwindigkeiten mehr oder weniger genau gemessen. Erste Angaben über Maximaldrücke stammen aus Russland aus den Jahre 1936-1939 [3]; seit 1952 sind auch in der Schweiz verschiedene Meßstellen gebaut worden, die schon viele interessante Ergebnisse lieferten [4]. Doch gestatten diese Anlagen kein systematisches Experimentieren mit bewegtem Schnee. Um dies zu ermöglichen, wurde am Eidg. Institut für Schnee- und Lawinenforschung in den Jahren 1954-1961 das nachfolgend beschriebene Projekt einer Schneegleitbahn entwickelt und verwirklicht. Damit wurde die Möglichkeit geschaffen, kïnstlich erzeugte und in vorgeschriebenen Bahnen gehaltene Schneebewegungen zu untersuchen ${ }^{2}$ ).

1) Die Ziffern in eckigen Klammern verweisen auf das Literaturverzeichnis, Seite 37 .

2) Es sei erwähnt, dass fast gleichzeitig wie in der Schweiz in Japan eine ähnliche Anlage gebaut wurds von der einige Ergebnisse bereits publiziert [5] vorliegen. 


\section{Generelles Messprogramm}

\subsection{Allgemeines}

Man teilt Lawinen bezüglich der Bewegungsform in Staub- und Fliesslawinen ein [6]. Der Übergang von der Fliessbewegung zur stiebenden Bewegung der Staublawinen (bei der die einzelnen Schneeteile in der Luft schweben) erfordert ziemlich hohe Geschwindigkeiten (minimal etwa $15-20 \mathrm{~m} \mathrm{sec}^{-1}$ ) und grosse Absturzhölıen. Der Vorgang gleicht dann der Bewegung eines schweren (rases (Luft plus Schnee) in einem leichteren (Luft). Aus verständlichen Gründen musste darauf verzichtet werden, solche Lawinenformen in das Messprogramm aufzunehmen. Die folgenden Ausführungen beschränken sich daher auf Fliesslawinen, bei denen die einzelnen Schneeteilchen immer mehr oder weniger miteinander in Berührung bleiben. Im Vordergrund steht die Untersuchung von Wirkungen des rasch bewegten Schnecs auf irgendwie geartete, am Boden aber fest verankerte Hindernisse; wobei sich die Versuchsanordnungen meist aus Problemen des praktischen Lawinenverbanes ergeben.

\subsection{Modellgesetzi}

IBei Experimenten mit der Schneegleitbalm muss beachtet werden, dass wegen der beschränkten Abmessungen der Anlage nicht alle Versuche in den wirlilichen Cirössenverlöilnisen durchgeführt werden können und

als Modellmaterial in genügenden Mengen nur der oberhalb der (ileitbahn natürlich abgelagerte schnee zur Verfïgung steht.

Infolge der erstgenannten Tatsache wird matn ab und zu gezwungen sein, einen Verkleinerungsmaßstab zu wählen. Dic Unrechnung auf wirkliche Verhältnisse hat tann auf Grund von Kennzahlen zu geschelıen. Diı, wie schon erwähnt, sowohl Trägheits- wie Massen- und Reibungskräifte auftreten, sind dic Vorgänge durch folgende 5 Grössen bestimmt: Charakteristische Geschwincligkeit $U$, charakteristische Länge $L$, Dichte $\varrho_{0}$, Zähigkeit $\mu$ und Erdbeschleunigung $g$. Daraus lassen sich zwei voneinander unabhängige Kenngrössen ableiten [7], nämlich die Revnoldssche $\left(R e=\varrho_{0} U L / \mu\right)$ und lie Froudesche $(F r=U / V g L)$ Zahl.

Um eine vollkommene Ähnlichkeit zu erhalten, miissten beide Kennzahlen eingeaalten werden. Dies ist infolge des vorgegebenen Modellmaterials Schnee nicht mögich, was kurz erläutert werden soll. Bezeichnen wir die Grössen in der Natur mit einem ndex $N$, diejenigen im Modell mit Index $M$, so transformieren sich die Cieschwindigseiten mit

$$
y=I_{y}
$$

xei gegebenem Modellmaßstab

$$
\lambda=\begin{aligned}
& I_{y} \\
& I_{y y}
\end{aligned}
$$

ach der Reynoldsschen Bedingung $\left(v=\prime \prime / \underline{Q}_{0}\right.$ kinematische Zähigkeit) gemäss

$$
\varepsilon_{K F}=\begin{array}{ll}
1 & r_{X} \\
\lambda & r_{11}
\end{array}
$$


nach der Froudeschen Bedingung (bei Gleichsetzung der Erdbeschleunigungen in Natur und Modell) gemäss

$$
\varepsilon_{F r}=\lambda^{1 / 2} .
$$

Bei gleichzeitiger Erfüllung beider Bedingungen entsteht die Forderung

$$
\lambda=\left(\frac{r_{N}}{r_{M}}\right)^{2 / 3} \text {. }
$$

Mit Schnee als Modellmaterial wird $v_{N} / v_{M}=1$ und damit der allein zulässige Modellmaßstab $\lambda=1$.

Es besteht aber trotzdem eine Möglichkeit, den Einfluss beider Ähnlichkeitszahlen zu kontrollieren, indem nämlich derselbe Versuch in verschiedenem Modellmaßstab und mit konstant gehaltener $F r$-Zahl durchgeführt wird. Weil so die Re-Zahl ändert, erhalten wir deren Einfluss auf eine interessierende Grösse. (Der umgekehrte Weg, konstant gehaltene Re-Zahl und variable Fr-Zahl, kommt wegen der erforderlichen, gegenüber der Natur erhöhten Geschwindigkeiten nicht in Frage.) Ein grosser Bereich von $R e$ kann allerdings zufolge der beschränkten Möglichkeiten in der Wahl des Modellmaßstabes nicht erfasst werden $\left[R e=L^{3 / 2} \operatorname{Fr} g^{1 / 2} \gamma^{-1}\right]$.

\subsection{Druckwirkungen}

Den wichtigsten Problemkreis der Schneegleitbahn umfasst die Druckwirkung von fliessendem Schnee auf ein irgendwie geartetes Hindernis [8]. Wenn Schnee gegen ein solches zufliesst, kann der sich abspielende Vorgang in zwei Phasen unterteilt werden. In der ersten Phase verursacht der zuerst auftreffende Schnee einen zeitlich veränderlichen Stossdruck. Bleibt der Schneezufluss nachher eine gewisse Zeit lang erhalten, stellt sich die zweite Phase mit einem mehr oder weniger zeitunabhängigen Strömungswiderstand ein. Die Vorgänge der ersten Phase lassen sich rechnerisch wohl nur in einfachsten Fällen überblicken. Abschnitt 5.1 dieser Arbeit enthält einen ersten Versuch dazu. Die Berechnung des konstanten Strömungswiderstandes ist für gewisse Hindernisse sehr einfach, muss aber im allgemeinen ebenfalls experimentell ergänzt werden.

Das Untersuchungsprogramm umfasst Hindernisse mit vollständig geschlossener und solche mit gegliederter Hindernisfläche. Erstere lassen sich in eine Gruppe aufteilen bei der durch eine ebene Stauwand, die in beiden Abmessungen senkrecht zur Anströmrichtung wesentlich grösser ist als der Anströmquerschnitt, sämtliche Schneeteilchen in zur Hindernisebene parallele Bahnkurven abgelenkt werden und in eine andere Gruppe, bei der die auch nichtebene (zum Beispiel keilförmige) Stauwand in einer solchen Abmessung klein ist, also umflossen werden kann. Bei Hindernissen der ersten Gruppe interessiert vor allem der zeitlich veränderliche Stossdruck, während die zweite Gruppe vorwiegend der Untersuchung von zeitunabhängigen Strömungswiderständen dienen soll. Unter gegliederten Hindernissen werden die im Lawinenverbau des Anbruchgebietes praktisch vorkommenden Verbauungswerke verstanden. Der Stossdruck soll dabei als Funktion der Deformationsfähigkeit und Konstrultionsart des Hindernisses untersucht werden und der stationäre Strömungswiderstand als solche der verschieden gestalteten Gliederung der Hindernisfläche.

Gemessen werden soll der Kraftverlauf sowohl in der Hindernisfläche wie an Auflagerungspunkten des Hindernisses. Vom auftreffenden Schnee soll die Schneeart 
(gemäss Schneeklassifikation), das Raumgewicht, clie Temperatur, die Geschwincligkeit vor dem Aufprall und die Fliesshöhe bekannt sein.

\subsection{Strömungsformen}

Mit den Druckwirkungen sollen auch die Strömungsformen untersucht werden. Bei diesen Versuchen ist man meistens gezwungen einen Modellmaßstab $\lambda>1 \mathrm{zu}$ wählen. Folgende Einflüsse sollen untersucht werden: Derjenige der konstruktiven Gestaltung einer gegliederten Fläche auf den Durchströmvorgang, der Hindernisform auf die Umströmung und schliesslich der Rauhigkeitsverhältnisse des Bodens auf eine Schneebewegung ohne Störung durch ein Hindernis.

\section{Aufbau der Schneegleitbahn}

\section{1. Übersicht}

Die Schneegleitbahn ist parallel zur Ostfassade des Eidg. Institutes für Schneeund Lawinenforschung auf Weissfluhjoch ob Davos (2665 m ï. M.) angeordnet. Sic besteht im wesentlichen aus einer $20 \mathrm{~m}$ langen und $2,5 \mathrm{~m}$ breiten Beschleunigungsstrecke, die in ihrem obersten Teil einen Schneesammler enthält, sowie einer $3,5 \mathrm{~m}$ langen und 3,4 m breiten Meß 3 strecke. Damit die Geschwindigkeit variiert werden kann, wurde die Mögliclıkeit einer Neigungsänderung der Beschleunigungsstrecke geschaffen. Die Meßstrecke ist in ihrer Neigung ebenfalls unabhängig variierbar, so dass eine Winkeländerung zwischen den beiden Strecken möglich ist. Der prinzipielle Aufbau der Anlage ist aus Figur 1 ersichtlich.

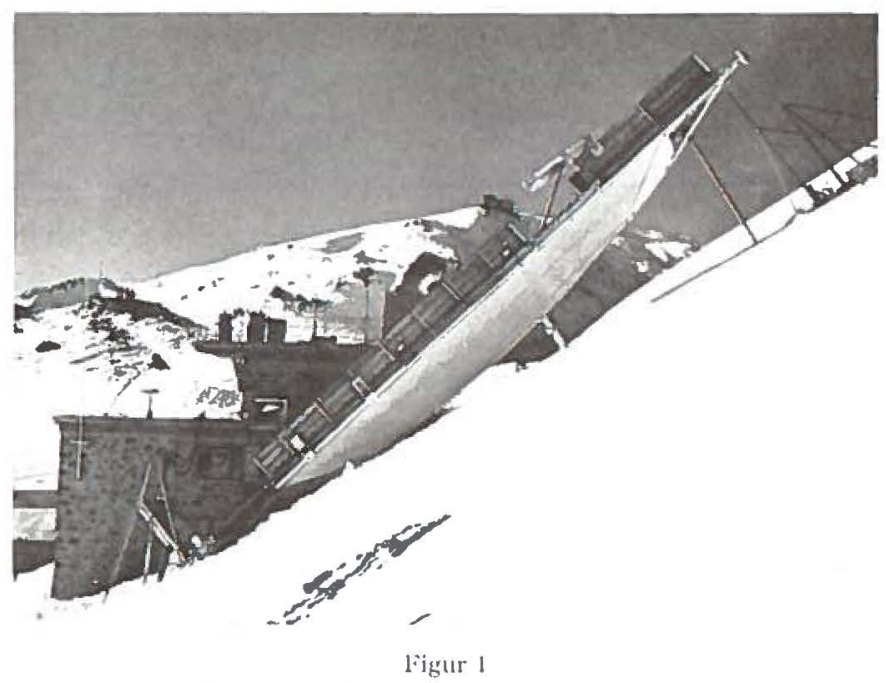

Übersichtsaufnalune der Schne'pleitbahn.

\subsection{Die Beschleunigungsstrecke}

Das Tragwerk der Beschleunigungsstrecke besteht in einer aus Rohren zusammengesetzten Dreigurt-Fachwerkkonstruktion. Als Konstruktionsmaterial wurde hierzu 
Aluminium "Unidal 43" verwendet [9]. Die Beschleunigungsstrecke ist auf der Talseite gelenkig gelagert; auf der Bergseite ruht sie auf einer pendelstützenartig ausgebildeten, hydraulischen Hebevorrichtung. Diese weist bei einem Totalhub von $4,1 \mathrm{~m}$ (Hebezeit 17 Minuten) eine maximale Tragkraft von 17,1 t auf. Diese Vorrichtung erlaubt es, eine rasche Neigungsänderung in einem Winkelbereich von $35-45^{\circ}$ auszuführen. Der Aluminiumbelag, auf dem der Schnee gleitet, besteht aus sogenannten Muraliprofilen. Seitlich ist die Beschleunigungsstrecke durch $1 \mathrm{~m}$ hohe, abklappbare Holzwände abgegrenzt um ein seitliches Ausfliessen von kohäsionsarmen Schneearten zu verhindern.

Der Schneesammler im obersten Teil der Gleitbahn dient zur Bereitstellung des zu einem bestimmten Versuch notwendigen Schnees, er ist in Richtung Meßstrecke mit einer elektrisch auslösbaren Absperrklappe versehen. Damit diese den Startvorgang des Schnees durch ihr Eigengewicht nicht hemmt, wurde sie mit einem Gegengewicht ausbalanciert. Die Länge des Schneesammlers kann durch freie Wahl der Lage der Absperrklappe beliebig festgelegt werden. Vorläufig wurde sie $5 \mathrm{~m}$ lang gewählt, so dass maximal 12,5 $\mathrm{m}^{3}$ Schnee eingefüllt werden können. Das Einfüllen geschieht üblicherweise von Hand mit Schnee der unmittelbaren Umgebung. Es ist aber auch möglich, den Sammler einschneien zu lassen, man hat dann einen natürlichen und nicht durch Schaufeln irgendwie veränderten Versuchsschnee. Diese letztere Methode wurde aber bis jetzt deshalb nicht angewendet, weil sie Reihenversuche praktisch ausschliesst.

\subsection{Meßstrecke}

Die Tragkonstruktion besteht aus sieben in Achsabständen von etwa $0,5 \mathrm{~m}$ parallel zur Fallinie angeordneten DIE-Trägern und aus je zwei Quer- und Diagonalträgern. Oben ist die Meßstrecke gelenkig und in der Fallinie verschieblich aufgelagert. Der untere Querträger ruht auf einer hydraulischen Hebevorrichtung, welche eine rasche Neigungsänderung im Bereich $30-45^{\circ}$ erlaubt. Die Hebevorrichtung besitzt eine Tragkraft von $11 \mathrm{t}$ und einen Totalhub von $0,9 \mathrm{~m}$.

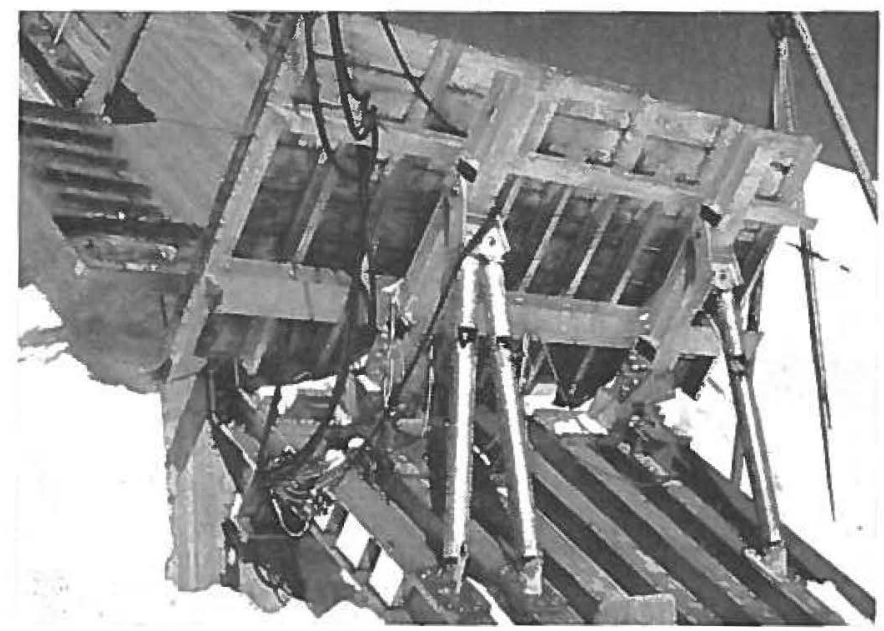

Figur 2

MeBstrecke mit Stauwand. 
Auf der Meßstrecke sollen alle interessierenden Hindernisse aufgebaut werden können. $\mathrm{Zu}$ diesem $Z$ weck wurde eine Grundausrüstung konstruiert, aus der sich jede Versuchsanordnung baukastenmässig zusammensetzen lässt. Diese Ausrüstung bcsteht im wesentlichen aus zwei Böcken, die sich aus einem Träger, an dem weitere Bauelemente befestigt werden, und einer oder zwei Stützen zusammensetzen. Die Träger können entweder senkrecht zur Meßstrecke oder in 3 Stufen $15^{\circ}, 30^{\circ}$ und $45^{\circ}$ zur Senkrechten geneigt, aufgestellt werden.

\section{Messmethoden}

\subsection{Die Krïftemessung}

Für die líräftemessung sollen nur kleine Wege (möglichst starre Hindernisse) zugelassen werden. Im weiteren sollen relativ hohe Frequenzen (bis etwa $2 \mathrm{kHz}$ ) erfassbar sein. Diese beiden Forderungen können am besten durch die Anwendung von Dehnungsmeßstreifen erfüllt werden. Für die Messung der Auflagerreaktionen an den Böcken wurden deshalb Philips Druckkraftgeber PR 9226 mit einer Nennlast von $10 \mathrm{t}$ bei den Trägerfüssen und einer solchen von 20) t in den Stützen, verwendet. Die Deformation (les Druckstabes im Geber betriigt bei Nennlast weniger als $0,1 \mathrm{~mm}$, der Höchstwert der erfassbaren lirequenzen liegt bei etwa $100 \mathrm{kHz}$.

Für die Messung von cinzelnen Reaktionen in den Hindernisflächen (zum Beispiel spezifischer Druck oder Auflagerkraifte von Konstruktionselementen) wurde von unserem Institut eine den spezicllen Anforderungen entsprechende Druckdose entwickelt. Diese Dose erlaubt mit Hille von auf Biegeplättchen aufgeklebten Dehnungsmeßstreifen die Messung von Normal- wie Querkräften. Die Grösse der Nennlast kann dabei durch auswechseln der Biegeplättchen auf eine gewünschte Grösse (bis $1 \mathrm{t}$ ) gebracht werden.

Die Druckdosen sind über abgeschirmte, etwa $30 \mathrm{~m}$ lange 10 -fach-Kabel mit dem Messraum verbunden.

Weil an den Hindernissen auch simultan an mehreren Stellen gemessen werden soll, wird das Signal eines Gebers vorerst auf cinen Quecksilber-Umschalter gegeben, der die Meßstelle mit einer Frequenz von 50 Umdrehungen pro Sekunde abtastet. Der Schalter besitzt 64 Kontakte. Die zu registrierenden Vorgänge verlangen aber, wie Versuche gezeigt haben, einige hundert Messpunkte pro Sekunde und Meßstelle (Breite einer Druckspitze etwa $+\mathrm{ms}$ ). Somit kann nicht jedem Kontakt eine Meßstelle zugeordnet werden. Bei 8-facher Schaltung von 8 Meßstellen resultiert ein Zeitintervall von 2,5 ms zwischen den Messpunkten, was ein «Durchschlüpfen» der Druckspitze verhindern sollte.

Für die Registrierung der Kräfte wird ein Kathodenstrahloszillograph verwendet. Eine unmittelbar vor dem Hindernis aufgestellte Photozelle (siehe Abschnitt 4.2) löst kurz vor dem Aufprall des Schnees die Zeitablenkung aus.

Als Ergänzung zu den beschriebenen elektrischen Druck-Messungen werden noch mechanische vorgenommen. Dazu wird eine von unseren Institut für Lawinendruckmessungen in der Natur angewendete Druckdose übernommen. Das Messprinzip ist ähnlich dem der Härteprüfung nach Vickers, indem eine Kreiskegelspitze aus Stahl in ein Raffinalplättchen eingedrückt wird. Mit dem Durchmesser des entstandenen Eindruckes kann an Hand einer Eichkurve auf die ausgeübte Kraft geschlossen werden. 
Genaue Resultate können mit dieser Messart nicht erwartet werden, sie wurde vielmehr eingeführt, um Aufschluss über die Frage $\mathrm{zu}$ erhalten, ob die mit statisch wirksamen Kräften aufgenommene Eichkurve auch einigermassen für kurzzeitige Belastungen gilt.

\subsection{Die Geschroindigkeitsmessung}

Zur Geschwindigkeitsmessung dienen längs der Beschleunigungsstrecke angeordnete Lichtschranken. Mit diesen können nur die translatorische Frontgeschwindigkeit des Versuchsschnees und bei Zerfall des ursprünglichen Blockes in mehrere Einzelblöcke evtl. auch die Translationsgeschwindigkeiten der nachrutschenden Massen erfasst werden. Die bei vielen Lawinentypen beobachtete kompliziertere Fliessbewegung, bei der die Frontgeschwindigkeit geringer ist als die der nachfliessenden Hauptmassen, und bei der die einzelnen Schollen zusätzlich noch eine Rotationsbewegung ausführen, kann nicht ausgemessen werden. Dies ist jedoch nicht nötig, da die Schneeteilchen auf der Beschleunigungsstrecke (bisherige Versuche haben das bestätigt) ohne wesentliche Relativgeschwindigkeiten zueinander, rein translatorisch abgleiten ${ }^{3}$ ).

Im einzelnen geschieht die Geschwindigkeitsmessung auf folgende Weise: Auf der Beschleunigungsstrecke sind je 4 Lichtwerfer und Photozellen am Tragwerk montiert. Der durch den Lichtstrahl des ersten Lichtwerfers durchgehende Schnee deckt die erst $€$ Photozelle der ersten Meßstrecke ab und setzt ein erstes Zählwerk in Gang. Dieses wird im Moment des Durchganges vor der zweiten Photozelle stillgesetzt, gleichzeitig aber wird das zweite Zählwerk eingeschaltet. Dieser Vorgang wiederholt sich über all 3 Meßstrecken bis bei der letzten Photozelle nur das dritte Zählwerk abgeschaltet, zu* sätzlich aber die Zeitablenkung des Oszillographen für die Druckmessung ausgelöst wird.

Das Zeitmessgerät erreicht die ausreichende Genauigkeit von etwa 1,5 ms.

Wegen des meist blockartigen Abgleitens der Schneemassen wird eine Sperrun€ der einzelnen Photozellenstrecken nach dem ersten Durchgang vorgenommen. Dit Sperrzeit beträgt etwa $25 \mathrm{sec}$. Damit die Zählwerke nicht durch kleinere, der eigent lichen Front vorauseilende, für die Druckwirkung aber nicht massgebende Scholler ausgelöst werden, sind die Lichtschranken in einer Höhe von $13-30 \mathrm{~cm}$ über den Gleitbelag verschiebbar angebracht. Die jeweilige Einstellung dieses Wertes muss au: der Versuchserfahrung gewonnen werden.

\section{Bisherige Resultate und Diskussion}

\subsection{Angenäherte theoretische Betrachtung des Stossvorganges}

Aus früheren Arbeiten [10] [11] ist bekannt, dass sich das mechanische Verhalte1 von kohärentem Schnee recht gut durch einen Burgers-Körper [12] darstellen lässt Die Deformation dieses Körpers beträgt

$$
x=P\left[\frac{1}{E_{1}}\left(1+\frac{t}{\tau_{1}}\right)+\frac{1}{E_{2}}\left(1-\exp \frac{-t}{\tau_{2}}\right)\right],
$$

3) Wenn wirklichkeitsnähere Fliessbewegungen reproduziert werden sollen, besteht die Möglichkeit di Rauhigkeit des Gleitbelages zu erhöhen und zugleich die Beschleunigungsstrecke einschneien zu lassen. 
wo $t$ die Einwirkzeit einer Belastung $P, E_{1,2}$ die Elastizitätsmoduli und $\tau_{1,2}$ die $\operatorname{Re}$ laxationszeiten bedeuten. Weil nun die Relaxationszeiten von Schnee in der Grössenordnung von Minuten liegen, vereinfacht sich die Deformationsgrösse auf

den rein elastischen Anteil.

$$
x=\frac{P}{E_{1}},
$$

Wir gehen von einem homogenen prismatischen Schncestück mit einem Querschnitt $F$ und einer Länge $l$ aus. Es bewege sich mit einer einheitlichen Geschwindigkeit vom Betrage $v_{0}$ auf ein senkrecht zur Bewegungsrichtung steherides ebenes nicht starres Hindernis zu, dessen Fläche im Verhältnis zu F gross ist. Die durch das Eigengewicht des Schnees auf die Wand ausgeübten Kräfte werden wegen ihrer Kleinheit (s. Abschnitt 5.2) im folgenden immer vernachlässigt.

Beim Aufprall führt das Schneeprisma, ähnlich wie ein elastischer Körper, Schwingungen aus. Da jedoch Schnee Merkmale einer Flüssigkeit hat und daher keine Formbeständigkeit besitzt, werden die in Wandnähe liegenden Schneepartikel in zur Wandebene parallele Bahnkurven abgedrängt. Das im Moment des Aufpralles vorhandene Prisma verliert so seine Form. Dic Schwingungen werden abklingen und nach einiger Zeit wird sich, sofern $l$ nicht $z u$ klein ist, ein mehr oder weniger stationärer Zustand einstellen. Der Einfachheit halber soll hier nur das Anfangsstadium kurz nach dem Aufprall (Schnee als elastischer Körper) und das stationäre Endstadium (Schnee als Flüssigkeit) untersucht werden. Der komplizierte Úbergang zwischen beiden Stadien ist von untergeordneter Bedeutung. Das Schneeprisma soll im Anfangsstadium als eindimensionales elastisches liontinuum, für welches die Differentialgleichung

$$
c^{2} \frac{\partial^{2} u}{\partial x^{2}}=\frac{\partial^{2} u}{\partial t^{2}}
$$

gilt, betrachtet werden. ( $c=V E / \varrho$ ist die Schallgeschwindigkeit und $u(x, l)$ dic Verschiebung eines Querschnittes). Dank der Tatsache, dass die Poissonschen Zahlen für Schnee im allgemeinen in der Nähe von null liegen [13], darf diese Annahme für die in allen Abmessungen gleiche Grössenordnung aufweisenden Schneeprismen getroffen werden. Das Hindernis wird vereinfacht durch eine Masse $M$ und eine Feder mit der Konstanten $f$ dargestellt (Figur 3)

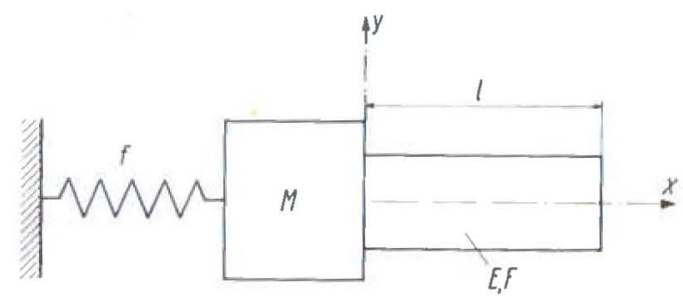

Figur :3

Annabule zur Stossdruckberechnung.

Dic Rand- und Anfangsbedingungen lauten:

$$
\left(\frac{\partial u}{\partial x}\right)_{x=1, t}=0
$$


beziehungsweise

$$
\left(E F \frac{\partial u}{\partial x}-u f-M \frac{\partial^{2} u}{\partial t^{2}}\right)_{x-0, t}=0,
$$

$$
\begin{aligned}
& u_{x, t=0}=0 \text {, } \\
& \left(\frac{\partial u}{\partial t}\right)_{x, l=0}=\left\{\begin{array}{ccc}
-v_{0} & \text { für } & 0<x \leqq l \\
0 & \text { für } & x=0,
\end{array}\right.
\end{aligned}
$$

wobei die Schreibweise der letzten Beziehung das Auftreten von unendlich grossen Kräften verhindern wird.

Das Lösungsverfahren nach BeRnoulLi [14] eignet sich für den vorliegenden Fall nicht. Wenn die Beziehungen mit den neuen dimensionslosen Veränderlichen

$$
t^{*}=\frac{c l}{l}, \quad x^{*}=\frac{x}{l}, \quad u^{*}=\frac{u c}{v_{0} l}
$$

geschrieben werden, erhält man

$$
\begin{gathered}
\frac{\partial^{2} u^{*}}{\partial x^{* 2}}=\frac{\partial^{2} u^{*}}{\partial t^{* 2},} \\
\left(\frac{\partial u^{*}}{\partial x^{*}}\right)_{x^{*}=1, t^{*}}=0, \\
\left(x_{1}^{2} \frac{\partial u^{*}}{\partial x^{*}}-u^{*}-x_{2}^{2} \frac{\partial^{2} u^{*}}{\partial t^{* 2}}\right)_{x^{*}-0, t^{*}}=0, \\
u_{x^{*}, t^{*}=0}=0, \\
\left(\frac{\partial u^{*}}{\partial t^{*}}\right)_{x^{*}, t^{*}-0}=\left\{\begin{array}{rll}
-1 & \text { für } 0<x^{*} \leqq 1 \\
0 & \text { für } x^{*}=0,
\end{array}\right.
\end{gathered}
$$

wo $\varkappa_{1}^{2}=E F / f l$ und $\varkappa_{2}^{2}=M c^{2} / f l^{2}$ dimensionslose positive Grössen sind. Die LaplaceTransformation

$$
U(p, x)=\int_{0}^{\infty} e^{p t} u(t, x) d t
$$

führt - die neuen Veränderlichen im weiteren ohne Stern geschrieben - auf die gewöhnliche Differentialgleichung

$$
\begin{aligned}
& \frac{d^{2} U}{d x^{2}}-p^{2} U=\left\{\begin{array}{lll}
1 & \text { für } & 0<x \leqq 1 \\
0 & \text { für } & x=0
\end{array}\right\}=f(x), \\
& \left(\frac{d U}{d x}\right)_{x=1, t}=0, \\
& \left(x_{1}^{2} \frac{d U}{d x}-U\left(1+\varkappa_{2}^{2} p^{2}\right)\right)_{x=0, t}=0
\end{aligned}
$$

deren Lösung

$$
U_{(x, p)}=\frac{\cosh (p x)-1}{p^{2}}+A e^{p x}+B e^{-p x}
$$


lautet, wobei

und

$$
\begin{aligned}
& A=C\left(1+x_{1}^{2} p+x_{2}^{2} p^{2}\right), \\
& B=-C\left(1-x_{1}^{2} p+x_{2}^{2} p^{2}\right)
\end{aligned}
$$

$$
C=-{ }_{2}^{2} p^{2}\left[\left(1+x_{1}^{2} p+x_{2}^{2} p^{2}\right) e^{p}+\left(1-x_{1}^{2} p+x_{2}^{2} p^{2}\right) e^{-p}\right]
$$

ist. Da vorläufig nur die Kr räfte in $x=0$ interessieren, braucht die Rücktransformation nur für den Ausdruck

$$
\left(\frac{\partial U}{\partial x}\right)_{10}=-\frac{1}{p\left[\operatorname{cotgh} p+\frac{x_{1}^{2} p}{1+x_{2}^{2} p^{2}}\right]}
$$

ausgeführt zu werden. Diese gelingt leicht mit dem Residuensatz, wenn beachtet wird. dass die Pole auf der imaginären Achse liegen müssen, weil ein reeller Anteil eine hier nicht vorausgesetzte Dämpfung zur Folge hätte.

Es gilt, wenn mit $J_{1}$ das Integral über den ganzen Weg und mit $J_{2}$ dasjenige über den Halbkreis bezeichnet wird (Figur 4)

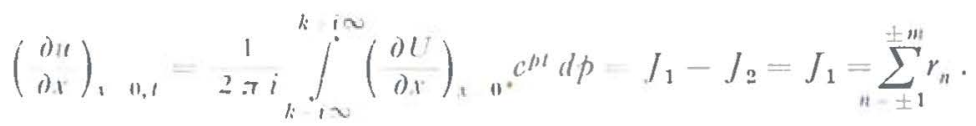

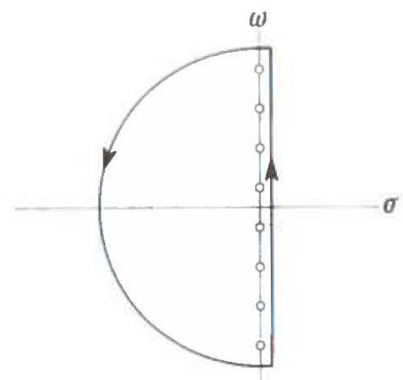

ligur 4

Iutegrationsweg.

Die Pole gewinnt man $(p=0$ ist kein Pol) aus

$$
\operatorname{cotg} \omega_{n}=1 \frac{\varkappa_{1}^{2} \omega_{n}}{1-\varkappa_{2}^{2} \omega_{n}^{2}}
$$

und die Residuen aus

$$
r_{n}=\frac{c^{n t}}{d p}\left[p\left(\operatorname{cotgh} p+\frac{x_{1}^{2} p}{1+x_{2}^{2} p^{2}}\right)\right] p i_{n}
$$

Zu

$$
r_{n}=\underset{\sin ^{2} \omega_{n}}{\omega_{n}}-\operatorname{cotg} \omega_{n}+\frac{2 x_{1}^{i \omega_{n} t} \omega_{n}}{\left(1-\varkappa_{2}^{2} \omega_{n}^{2}\right)^{2}} .
$$


Schliesslich ist

$$
r_{n}+r_{-n}=\frac{2 \sin \left(\omega_{n} t\right)}{\frac{\omega_{n}}{\sin ^{2} \omega_{n}}-\operatorname{cotg} \omega_{n}+\frac{2 \varkappa_{1}^{2} \omega_{n}}{\left(1-\varkappa_{2}^{2} \omega_{n}^{2}\right)^{2}}}
$$

und der Druck in $x=0$ (Stossdruck vor dem Hindernis $p_{s v}$ ), wenn wieder die ursprünglichen Bezeichnungen (siehe (8)) eingeführt werden und der Ausdruck etwas umgeformt wird

$$
p_{s t}=-2 \sqrt{E \varrho} v_{0} \sum_{n=1}^{\infty} \frac{\sin \left(\omega_{n} \begin{array}{l}
c \\
l
\end{array}\right)}{\omega_{n}\left(\operatorname{cotg}^{2} \omega_{n}+1\right)+\operatorname{cotg} \omega_{n} \frac{1+x_{2}^{2} \omega_{n}^{2}}{1-x_{2}^{2} \omega_{n}^{2}}} .
$$

Ist das Hindernis vollständig starr $(M$ oder $f \rightarrow \infty)$ wird

$$
p_{s, 1}=-\sqrt{E} \varrho v_{0} \frac{4}{\pi} \sum_{n}^{\infty} \frac{\sin \left(\frac{2 n-1}{2 l} \pi c t\right)}{2 n-1} \text {, }
$$

also rechteckförmig mit der Amplitude $V E \varrho v_{0}$ und der Periode $4 l / c$, wobei aber Zugkräfte ausgeschlossen sind, und daher, wenn der Schnee seine Gestalt beibehalten würde, nur die erste Hälfte der Periode auftreten könnte. Mit dem Satz vom Antrieb kann dieses Ergebnis sehr leicht kontrolliert werden.

Der Druckverlauf am nachgiebigen Hindernis unterscheidet sich von demjenigen am starren nur durch die in der Nähe von $1 / \varkappa_{2}$ liegenden Pole. Die rechteckförmige Verteilung wird dadurch etwas korrigiert und zwar umso mehr je grösser $\varkappa_{2}$ ist. $d p_{s v} / d t$ für $t=0$ ist für alle Fälle unendlich gross und der Anfangsdruck unabhängig von den Hinderniseigenschaften immer gleich $\sqrt{E \varrho} v_{0}$.

Bei den in 5.2 beschriebenen Versuchen wurden die Kräfte hinter der Masse des Messbockes gemessen. Aus der Differentialgleichung ( $x$ ist die Koordinate der Masse $M$ )

$$
\frac{d^{2} x}{d t^{2}}+K^{2} x=\frac{F}{M} p_{s t}
$$

und den zugehörigen Anfangsbedingungen folgt der Stossdruck hinter der Masse $p_{s h} z u$

$$
p_{s h}=-2 \sqrt{E \varrho} v_{0} \sum_{n=1}^{\infty} \frac{\sin \left(\omega_{n} c t\right)-\frac{\omega_{n} c}{K l} \sin (K t)}{\left(1-\omega_{n}^{2} \varkappa_{2}^{2}\right)\left[\omega_{n}\left(\operatorname{cotg}^{2} \omega_{n}+1\right)+\operatorname{cotg} \omega_{n} \frac{1+\varkappa_{2}^{2} \omega_{n}^{2}}{1-\varkappa_{2}^{2} \omega_{n}^{2}}\right]},
$$

wo

bedeutet.

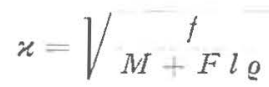

Schliesslich lässt sich der Ablenkdruck für das stationäre Endstadium aus dem Impulssatz zu

herleiten.

$$
p_{a}=\varrho v_{0}^{2}
$$


Wenn vorausgesetzt wird, dass der Anfangswert von $p_{s v}$ der in Wirklichkeit maximal auftretende Druck ist (s. Abschnitt 5.2), beträgt die Geschwindigkeit, für die dieser Stossdruck gerade gleich dem Ablenkdruck ist,

$$
v_{0}=\sqrt{\frac{E}{\underline{Q}}}=c \text {. }
$$

Im nächsten Abschnitt sollen die theoretischen Ergebnisse mit Versuchen verglichen und näher diskutiert werden.

\subsection{Im Winter 1960/61 durchgefïhrte Versuche}

Die im Winter 1960/61 durchgefïhrten Versuche dienten zur Abklärung des zeitlichen Kreisverlaufes auf eine ebene, relativ grosse, geschlossene Fläche. Die Hindernisebene wurde senkrecht zur ursprünglichen Bewegungsrichtung des Schnees angeordnet, so dass die Voraussetzungen der vorangegangenen Theorie erfüllt sind. Um den Stossvorgang möglichst gut zu erfassen, wurde ohne Quecksilberumschalter mit nur einer Meßstelle gearbeitet. Dazu wurde die am einen Trägerfuss angebrachte, hangparallele Kräifte messende $10 \mathrm{t}$-Druckdose gewählt. Ferner wurden drei mechanische Druckdosen (siehe Abschnitt 4.1) eingesetzt, welche auf der Stauwand montiert sind, also keine nennenswerte Masse vor sich haben. Zur Geschwindigkeitsmessung wurde nur die unterste Meßstrecke verwendet.

Aus den total durchgeführten 6 Versuchen wurden Mittelwerte der gemessenen (rrössen gebildet, der Elastizitätsmodul anhand von [11] auf $E=5 \cdot 10^{4} \mathrm{kgm}^{-2}$ geschätzt und daraus mit den Gleichungen (23), (24) und (26) die Druckverläufe berechnet (Figuren 5 und 6).

Vergleicht man diese berechneten mit den gemessenen Werten, so ist folgendes bemerkenswert:

- Ein erstes Druckmaximum tritt übercinstimmend nach ca. $10 \mathrm{~ms}$ auf; bei den meisten Versuchen ebenfalls ein zweites nach ca. 25 ms. (Bei den registrierten Kurven ist die Zeitachse nach links positiv).

- Die gemessenen Amplituden sind, vor allem die der zweiten Druckspitze, kleiner als die berechneten. Dies kann einmal darauf zurückgefülırt werden, dass das Schneeprisma schon kurz nach dem Aufprall an der Front durch seitliches Ausquetschen (siche Figur 7) Material verliert. Dadurch können sich vor allem die der ersten

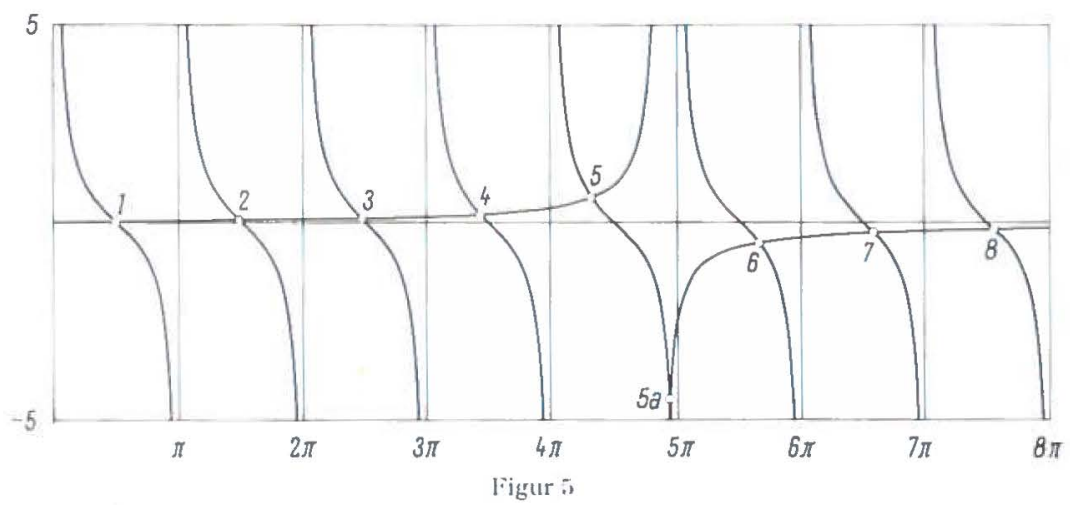

Craphische Bestimmung der Pole für $x_{1}^{2}=0,00966$ und $x_{2}^{2}=0,00130$. 


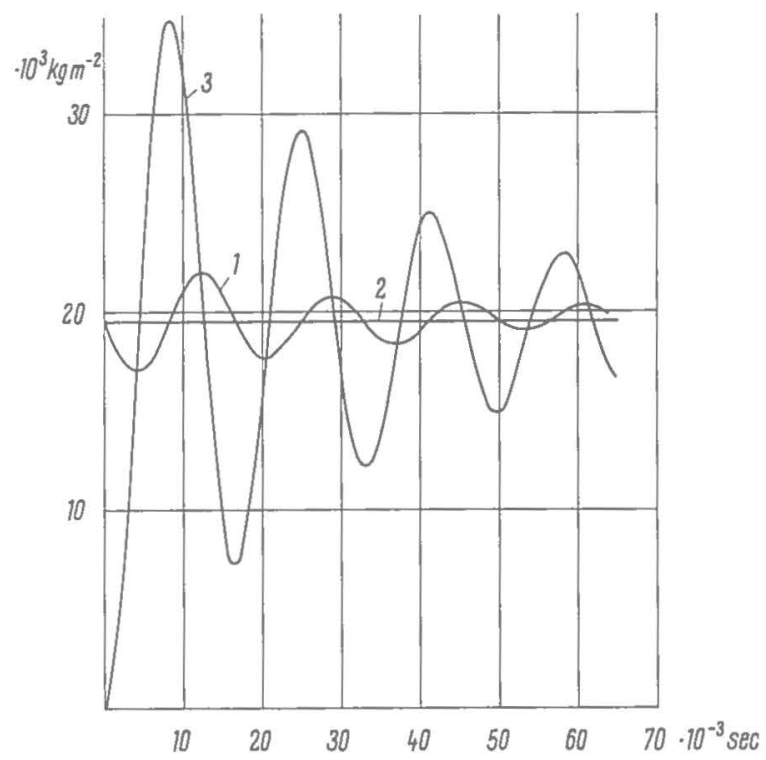

Figur 6

\%eitlicher Druckverlauf für $\varkappa_{1}^{2}=0,00966, \varkappa_{2}^{2}=0,00430, \varrho=51,8 \mathrm{~kg} \mathrm{sec}^{2} \mathrm{~m}^{-4}, E=510^{4} \mathrm{~kg} \mathrm{~m}^{-2}$ und $v_{0}=12,0 \mathrm{~m} \mathrm{sec}^{-1} ; 1$ vor der Masse, 2 vor einem starren Hindernis und 3 hinter der Masse.

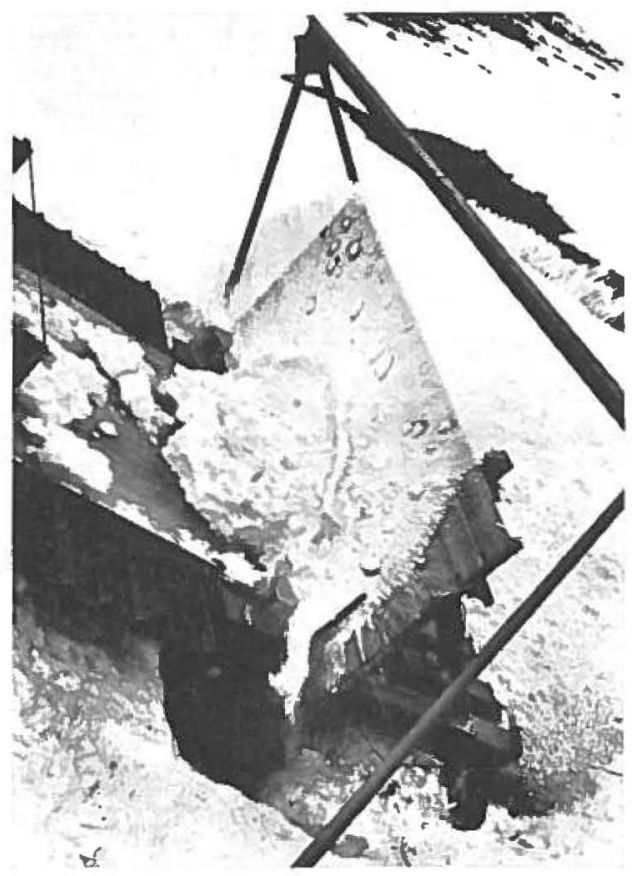

Figur 7

Versuchsschnee etwa 0,1-0,2 sec nach dem Aufprall. 
Versuchs- Bahn- Blockabmessungen nummer neigung nach dem Einfiullen und Natum
Länge Höhe Breite
Mittlere Endge- Elektrisch Mechanisch Dichte schwin- gemessener gemessener des ein- digkeit Maximal- Maximalgefüllten druck druck Schnees

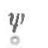

m

$\mathrm{m}$ $\begin{array}{llll}\varrho \mathrm{kg} & v_{0} & p_{8 m} & p_{\mathrm{s}}^{\prime \prime \prime} \\ \mathrm{sec}^{2} \mathrm{~m}^{-4} & \mathrm{~m} \mathrm{sec}^{-1} & 10^{3} \mathrm{~kg} \mathrm{~m} \mathrm{~m}^{-2} & 10^{3} \mathrm{~kg} \mathrm{~m} *\end{array}$

$53,30 \quad 12,09 \quad 4)$

16,62

5. 5.61

45

4,30

0,85

2,50

0,85

2,50

51,80

$1.2,23$

20,15

20,60

3. 5.6

$3 / 61$

23. 5. $61 \quad 45$

4,60

0,95

2,50

53,75

12,86

18,30

17,26

$1 / 61$

(1)

, 61

?. 6,61

4,72

$(1,92$

2,50

54,20

$\left.11,9^{5}\right)$

20,20

$17,1 \mathrm{~s}$

,$/ 61$

i. 6.61 4.5
0,98

2,50

45,00

11,90

15,00

$13,6.3$

4) Nicht gemessen, da Druckmaximum infolge Nullpunktverschiebung ausserhalb des Schirmes Iag.

i) Aus den Filmaufnahmen gemessen, da Geschwindigkeitsmessung nicht funktionierte.

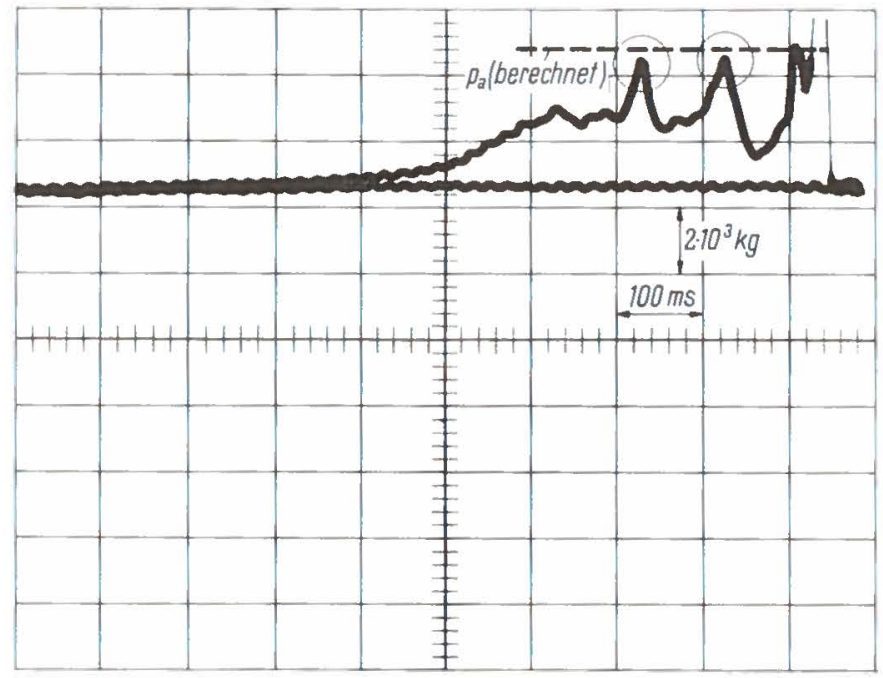

Zu Versuch 1/61. 


\begin{tabular}{|c|c|c|c|c|c|}
\hline $\begin{array}{l}\text { Zeit } \\
\text { bis zum } \\
\text { Druck- } \\
\text { maximum }\end{array}$ & $\begin{array}{l}\text { Ge- } \\
\text { schätztes } \\
\text { Elastizi- } \\
\text { tätsmodul }\end{array}$ & $\begin{array}{l}\text { Länge des } \\
\text { zuerst } \\
\text { auftref- } \\
\text { fenden } \\
\text { Blockes } \\
l \\
\mathrm{~m}\end{array}$ & $\begin{array}{l}\text { Bewegungsvorgang auf der } \\
\text { Beschleunigungsstrecke }\end{array}$ & $\begin{array}{l}\text { Statio- } \\
\text { närer } \\
\text { Ablenk- } \\
\text { cruck }\end{array}$ & $\begin{array}{l}\text { Verhältnis: } \\
\text { Maximaler } \\
\text { Stossdruck } \\
\text { zu statio- } \\
\text { närem Ab- } \\
\text { lenkdruck } \\
p_{s m} \\
p_{a}\end{array}$ \\
\hline 12,5 & 5 & 1,27 & $\begin{array}{l}\text { Zerfall in } 4 \text { Einzelblöcke. } \\
\text { Spalt nach dem ersten Block } \\
\sim 0,20 \mathrm{~m}\end{array}$ & 7,78 & 2,06 \\
\hline 9,2 & 5 & 1,24 & $\begin{array}{l}\text { Zerfall in } 3 \text { Einzelblöcke. } \\
\text { Spalt nach dem ersten Block } \\
\sim 0,20 \mathrm{~m}\end{array}$ & 7,75 & 2,60 \\
\hline 12,7 & 5 & 1,50 & $\begin{array}{l}\text { Block bleibt mehr oder } \\
\text { weniger kompakt. Spalt nach } \\
\text { dem ersten Block }(\sim 0,05 \mathrm{~m}) \\
\text { evtl. nicht durchgehend }\end{array}$ & 8,89 & 2,06 \\
\hline 15,4 & 5 & 1,06 & $\begin{array}{l}\text { Zerfall in } 4 \text { Einzelbiöcke. } \\
\text { Spalt nach dem crsten Block } \\
\sim 0,10 \mathrm{~m}\end{array}$ & 7,90 & 2,62 \\
\hline 9,6 & 5 & 1,30 & $\begin{array}{l}\text { Zerfall in } 4 \text { Einzelblöcke. } \\
\text { Spalt nach dem ersten Block } \\
0,25 \mathrm{~m} \text {. Nach dem zweiten } \\
\text { Block nur sehr kleiner Spalt }\end{array}$ & 7,67 & 2,63 \\
\hline 9,5 & 5 & 1,54 & $\begin{array}{l}\text { Zerfall in } 3 \text { Einzelblöcke, } \\
\text { wobci die beiden vordersten } \\
\text { Blöcke durch einen kleinen } \\
\text { Spalt von ca. } 0,05 \mathrm{~m} \text { ge- } \\
\text { trennt sind }\end{array}$ & 6,36 & 2,36 \\
\hline
\end{tabular}

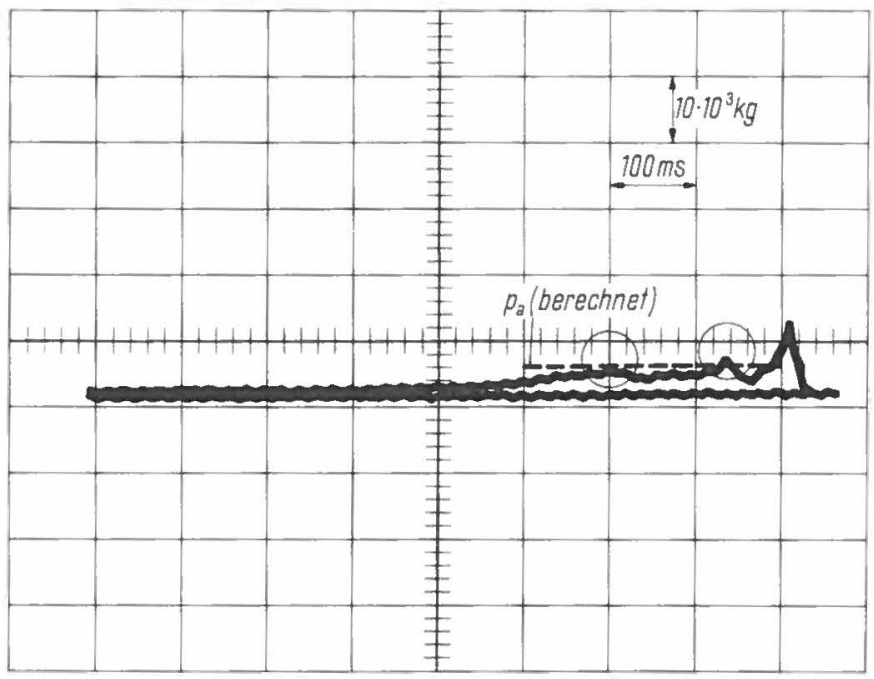

$\mathrm{Zu}$ Versuch 2/61. 


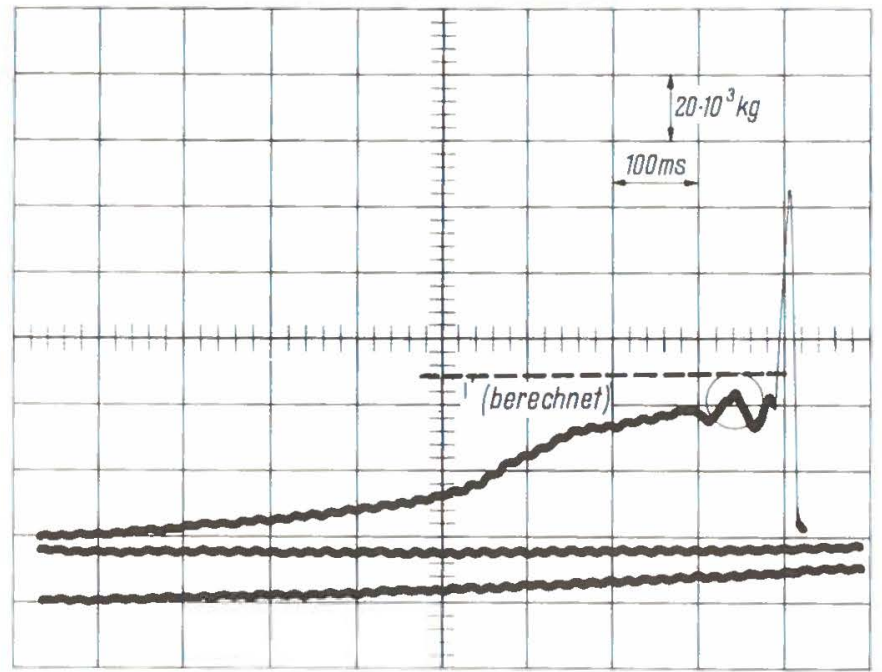

Zi1 Virsuch :3/61.

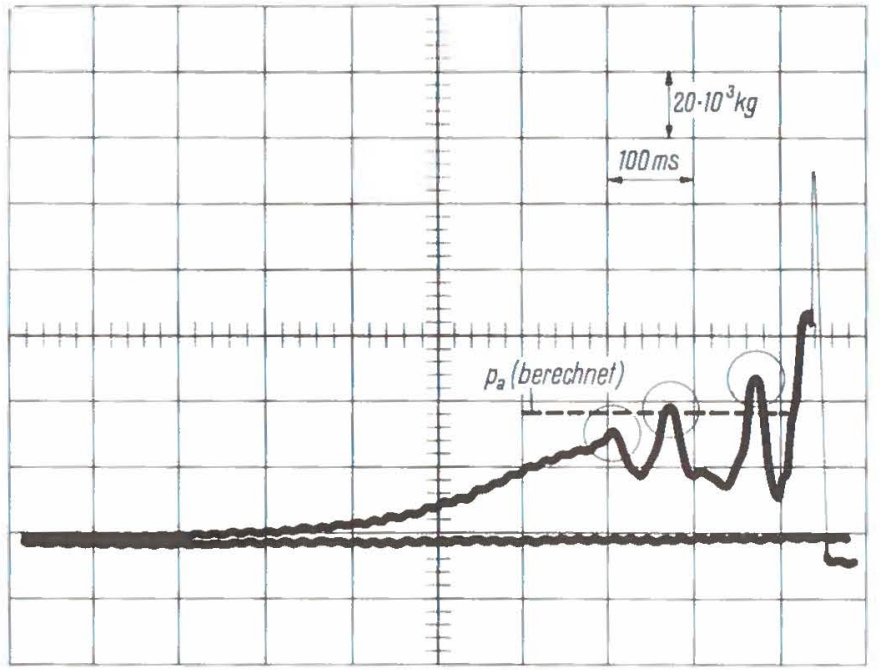

$Z$ Versuch $4 / 61$.

nachfolgenden Druckspitzen nicht mehr ausbilden, die zweite allerdings in vielen Versuchen noch knapp andeutungsweise. Dann ist ferner die Schätzung des Elastizitätsmoduls hauptsächlich für die Differenz der ersten Maxima verantwortlich zu machen. Um diese Schneecharakteristik genauer zu erfassen, müssen Laboratoriumsversuche mit einem dynamischen Oedometer ins Auge gefasst werden.

Die mechanische Druckmessung gibt gemäss Figur 6 eher zu grosse Werte, was auf die mindestens im statischen Versuch nachgewiesene Zunahme der Eindringtiefe mit der Einwirkzeit zurückgeführt werden kann. 


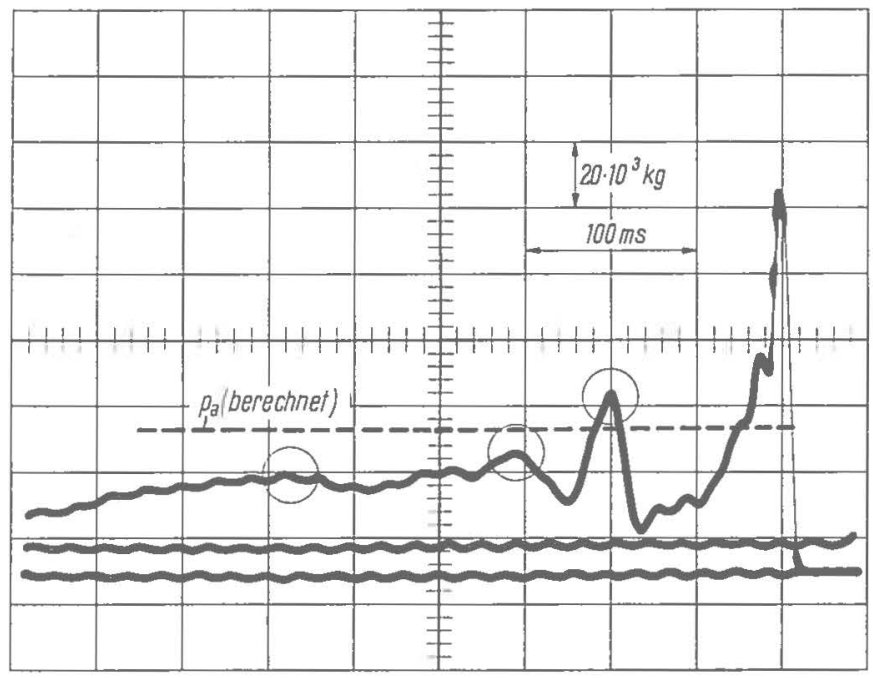

Zu Versuch $5 / 61$.

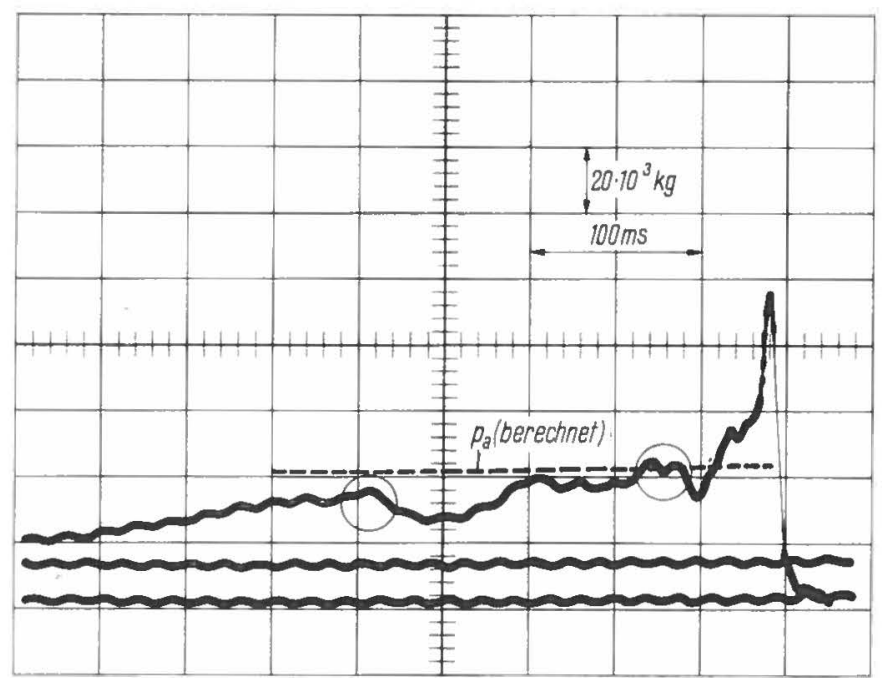

Zu Versuch 6/61.

- Ein stationärer Ablenkdruck ist in vielen Versuchen angedeutet.

- Es treten später noch weitere Druckmaxima auf, die mit dem ersten Aufprall nich mehr zusammenhängen (in den Diagrammen mit Kreisen bezeichnet). Filmauf nahmen beweisen, dass diese von nachfolgenden Blöcken stammen.

Die Versuche zeigen natürlich auch Unterschiede zwischen den tatsächlichen Ver hältnissen und den vereinfachenden oder idealisierenden Annahmen in der Theorie So kann das Hindernis in Wirklichkeit nicht wie in Figur 3 dargestellt werden, sodant spielt die Kompression der im Schnee eingeschlossenen Luft bei raschen Vorgänger 
auch eine gewisse Rolle und schliesslich stellt die Annalıme des Burgers-Körpers bei hohen Spannungen sicher nur eine grobe Annäherung dar.

\section{Nachwort}

Die Idee und das erste Messprogramm der Schneegleitbahn gehen auf Dr. M. DE Quervain, Direktor des Schnee- und Lawinenforschungsinstitutes zurück. An der Verwirklichung im Rahmen der Sektion Schneemechanik und Iawinenverbau haben zahlreiche ehemalige und heutige Mitarbeiter des Institutes mitgearbeitet, nämlich bei cler Planung und Beratung die Herren Ing. J. HARDEGger, Dr. C. JACCARD, dem der Verfasser für mathematische Ratschläge besonderen Dank schuldet, Ing. W. KENNeI., G. Kl.Ausegger, Dr. R. List und Ing. A. Roch und beim Bau und bei der Durchführung der Versuche die Herren H. HügI, H. P. Hürlemann, H. PAppa, E. SommerHALDER Und E. WENGI.

Verschiedene Firmen haben den Problemen dieses Projektes ihre besondere Aufmerksamkeit geschenkt; besondere Erwälmung verdient die sachkundige Beratung durch El. Ing. F. A. LoEschER bei der Lösung der elektronischen Probleme. Allen Beteiligten sei hier Dank und Ancrkennung ausgesprochen.

\section{ITTERITURVERZEICHNIS}

1] R. HALEEL, Schnemechanili mil Hinveisen auf die Erdbanmechanik, Beiträge zur Geologie der Schweiz-geotechnische Serie-Hydrologie, Lieferung 3 (1939).

[2] E. Bucher, Beitrag zu den theoretischen Grundlagen des Lawinenverbaus, Beiträge zur Geologie der Schweiz-geotechnische Serie-Hychrologie, Lieferung 6 (1948).

[3] A. G. Gors und G. F. OTres, Experimentelle Bestimmung der Einschlagskraft von Lawinen, Mitt, der Akademie für IVissenschaften cler UdSSR. Geographische und geophysische Serie Nr. 3, Moskau (1939).

[4] A. Roch, Mesure de la force des avalanches, in Schnee und Lawinen in den Schweizeralpen, Winter 1960/61, Winterbericht des Institutes SI.F Nr. 25, 124-136 (1962).

[5] I. Furukawa, Die Stosskraft von Larvinen, in Journal of the Japanese Society of Snow and Ice, Vol. 19 Nr. 5, September (1957).

[6] R. Haefeli, M. De Quervain, Gedanken und Anregugen zur Benennung und Einteilung von Lawinen, Die Alpen 31, $72-77$ (1955).

7] P. Grassmann, Physikalische Grundlagen der Chemic-Ingenienr-Technik (Verlag H. R. Sauerländer \& Co., Aarau und Frankfurt a. M. 1961).

[8] B. Salm, Messprogramm für die Schneegleitbahn, Int. Bericht 283 des Eidg. Institutes für Schnee- und lawinenforschung (1958).

[9] WV. Kennel, Statische Berechnung der Lawinengleitbahn, Int. Bericht 231 des Eidg. Institutes für Schnee- und Lawinenforschung (1957).

[10] M. de Quervain, Kristallplastische Vorgänge im Schneeaggregat II, Int. Bericht 24 des Eidg. Institutes für Schnee- und Lawinenforschung (19+6).

[11] 7. Yosida and Colleagues, Physical Studies on Deposited Snow 11. Mechanical Properties (1), Contributions from the Institute of Low Temperature Science, Hokkaido University, Sapporo, Japan (1956).

[12] M. Reinek, Rheology, In Handbuch der Physik Bd. V1, (Springer-Verlag, $472-474$ 1958).

[13] A. Roch, Discussion sur la valeur du nombre de Poisson m pour la neige, Int. Bericht 89 des Eidg. Institutes für Schnee- und Lawinenforschung (1948).

14] H. Ziegler, Mechanik 1 II (Birkhäuser Verlag 1952). 


\section{Summary}

A description of an installation is given that allows to observe and to measure artifi cially released masses of snow sliding over an inclined plane and especially to measure th impact pressure on an obstacle. The installation consists of an acceleration section 21 meters long and of a measuring section 3.5 meters long on which obstacles can be placed The forces are measured with strain gauges and for measuring the velocity of the moving snow masses light barriers are arranged along the acceleration section. The impact pressuri upon a yielding obstacle is calculated assuming an unidimensional elastic continuum.

(Eingegangen: 6. März 1964.) 